\title{
1. Perfiles de personas con deficiencia MENTAL BASADOS EN LAS FUNCIONES MOTRICES GRUESAS
}

\author{
Isabel M. ${ }^{a}$ Ferrándiz Vindel \\ Pilar Gútiez Cuevas \\ Facultad de Educación \\ Universidad Complutense de Madrid
}

\begin{abstract}
A pesar del escaso número de trabajos existentes, en los últimos años han ido apareciendo estudios que ponen de manifiesto la importancia que tiene la educación en el ámbito de los niños con deficiencia mental. A pesar de no tener alteraciones documentadas, algunos de estos niños tienen dificultades de movimiento significativas que les causan problemas a lo largo de su escolaridad.
\end{abstract}

A pesar de los efectos que provoca la incoordinación motriz gruesa (discinesias, sincinesias, apraxias, dispraxias), en general, se conoce poco de las características del funcionamiento motor grueso entre los deficientes mentales. Normalmente se les ha clasificado sobre la base de habilidades cognitivas (Speece, 1987; Swanson, 1988; Golger, Eliason y Richman, 1989; Swanson, Cochran y Ewens, 1990) y patrones de conducta socio-emocional (Speece, McKinney y Appelbaum, 1985; Fuerst, Fisk y Rourke, 1989). En cuanto a clasificar en el dominio motor, existen algunos estudios que han tenido en cuenta las habilidades motrices finas incluidas como una parte de la evaluación diagnóstica (Petrauskas y Rourke, 1979; Watson, Goldgar y Ryschon, 1983; Korhonen, 1991) ; o también se han clasificado grupos de niños con incoordinación motora, según funciones motrices finas y gruesas (Lyytinenn y Ahonen, 1988; Taylor, 1990; Hoare, 1994). Sin embargo, la relación entre deficiencia mental e incoordinación motriz aún no ha sido estudiada en profundidad como para elaborar una clasificación que permita realizar pro- 
gramas de intervención adecuados a cada uno de ellos, y, en general, han sido tratados separadamente.

Por ello, el propósito de este trabajo ha sido identificar posibles subtipos de alumnos con deficiencia mental, basados en el desarrollo de las funciones motrices gruesas, mediante técnicas de análisis de cluster (Speece, 1985; Gaviria Soto, 1986).

\section{MÉTODO}

\section{MUESTRA}

La muestra de este trabajo son alumnos que asisten a un centro concertado de Educación Especial de la Comunidad de Madrid. Del total de alumnos de este centro se han seleccionado 97 sujetos ( 42 chicos y 55 chicas), con una edad cronológica de 6 a 16 años, siguiendo una técnica de muestreo intencional o deliberado. Los criterios que se han considerado como requisitos necesarios para pertenecer a la muestra han sido:

1. Ser deficiente mental.

2. Tener un CI menor de 70, obtenido con las escalas WISC-R y/o MSCA.

3. Tener entre 6 y 16 años de edad.

4. No tener una deficiencia motórica asociada a la deficiencia mental, para evitar que su desarrollo motor grueso siga patrones (alterados) según su deficiencia motórica y no según su deficiencia mental.

\section{INSTRUMENTOS DE MEDIDA}

Tras analizar en profundidad los instrumentos existentes, y conforme a los trabajos consultados, se aplicaron las siguientes baterías:

- el «Tests Bruininks-Ozeretsky para medir la eficacia motriz» (BOTMP),

- la «Escala de Inteligencia Wechsler para niños. Revisada» (WISC-R),

- las «Escalas McCarthy de Aptitudes y Psicomotricidad para niños» (MSCA).

Se seleccionó el Bruininks-Ozeretsky para medir la eficacia motriz: 
- Por su amplia utilización en investigaciones realizadas sobre el área motriz en distintos ámbitos.

- Por ser de aplicación amplia (6 a 16 años de edad) con lo que se ha podido aplicar a toda la muestra.

- Por ser un instrumento para el diagnóstico de las habilidades motrices, disfunciones motrices y trastornos del desarrollo.

Este instrumento está compuesto por ocho subtests que evalúan aspectos motrices básicos como: agilidad, equilibrio, coordinación bilateral, fuerza, velocidad.

La batería completa proporciona cuatro estimaciones de la eficacia motriz:

1. Índice del funcionamiento motor grueso;

2. Índice del funcionamiento motor fino;

3. Índice completo de la batería (funcionamiento motor grueso y fino);

4. Índice de la forma abreviada de la batería para una breve visión de la eficacia motriz.

En el presente estudio, se analizó en profundidad el ámbito del funcionamiento del desarrollo motor grueso.

Asimismo, para valorar el grado de Deficiencia Mental, se utilizaron las siguientes baterías:

El WISC-R se ha consagrado como un instrumento de diagnóstico de gran utilidad en las áreas de evaluación educativa y de valoración de aprendizajes o de incapacidades.

El WISC-R está integrado por los mismos 12 subtests que la primera versión, construida en 1949, proporcionando tres estimaciones de la inteligencia general:

1. Cociente Verbal.

2. Cociente Manipulativo.

3. Cociente Total.

La MSCA permite obtener puntuaciones o índices de diferentes conductas cognitivas y motrices. La batería contiene 18 subtests independientes que 
evalúan las variables aptitudinales en las siguientes áreas: verbal, perceptivomanipulativa, numérica, memoria, motricidad, y general cognitiva o índice general intelectual.

\section{TRATAMIENTO DE LOS DATOS}

El objetivo fundamental de este trabajo es tratar de establecer las características generales del desarrollo motor grueso en los sujetos que presentan Deficiencia Mental y definir qué problemas presentan con mayor frecuencia.

La hipótesis general, base del presente estudio es:

"Es posible hablar de un desarrollo motor grueso característico en los sujetos con deficiencia mental".

Esta hipótesis general se puede subdividir en las siguientes hipótesis:

- Subhipótesis 1: Es posible obtener perfiles de desarrollo motor grueso en los deficientes mentales.

- Subhipótesis 2: Existe relación entre el nivel de desarrollo motor grueso y el grado de Deficiencia Mental.

A continuación detallamos cada hipótesis propuesta, definiendo en cada caso las hipótesis operativa, nula y alternativa, planteadas para su posterior contraste estadístico.

\section{Es posible obtener perfiles de desarrollo motor grueso en los deficientes mentales}

El problema fundamental que se plantea, en primer lugar, es saber si los perfiles de desarrollo motor grueso se pueden obtener con una fiabilidad suficiente en el individuo con Deficiencia Mental. Como hemos comentado anteriormente, se han obtenido perfiles de desarrollo motor con poblaciones con dificultades de aprendizaje (Miyahara, 1994) y con Deficiencia Mental (Arnaiz, 1983; Ariel, 1990; Pallisera, 1995), utilizando instrumentos de medida como el test de Bruininks - Ozeretsky (1972) o como la batería de Picq y Vayer (1977).

La hipótesis operativa planteada es «identificar subtipos homogéneos de sujetos con deficiencia mental, sin deficiencias motrices asociadas, según el rendimiento en habilidades motrices gruesas». Para poderla contrastar estadísticamente, planteamos las siguientes hipótesis: nula (Ho) y alternativa (H1): 
Ho: $\mu \mathrm{A}=\mu \mathrm{B}=\mu \mathrm{C}=\mu \mathrm{D}$

$\mathrm{H} 1: \mu \mathrm{A} \neq \mu \mathrm{B} \neq \mu \mathrm{C} \neq \mathrm{mD}$
La media obtenida por el grupo A (cluster 1) es igual a las medias obtenidas por los grupos B, C y D (clusters 2,3 y 4 ).

La media obtenida por el grupo A no es igual a las medias obtenidas por los grupos B, C y D.

Para validar esta hipótesis, los resultados obtenidos en los subtests del Bruininks-Ozeretsky fueron analizados estadísticamente con un «análisis de cluster», siguiendo los pasos de Speece (1985).

Se escogió este tratamiento, pues el análisis de cluster trata de localizar las agrupaciones «naturales» de los sujetos en función de sus similitudes o diferencias en todas las variables sobre las que se describe al grupo simultáneamente (Gaviria Soto, 1986, 165).

Dada una muestra de individuos, del que de cada uno se dispone de una serie de observaciones, el análisis de cluster sirve para clasificarlos en grupos lo más homogéneos posible en base a las variables observadas (Orden Hoz, 1985, 106).

Tras realizar estos análisis podemos decir que:

1. Es posible obtener perfiles de desarrollo motor grueso en una muestra de deficientes mentales, utilizando para ello el Test de BruininksOzeretsky.

2. Sobre las bases del análisis de cluster y el uso posterior de la técnica "post hoc" de Tukey, a partir del patrón del perfil y el número de sujetos en cada grupo, se juzgó que la solución de " 4 clusters" era la solución óptima, con vistas a producir los perfiles de desarrollo motor. Era la agrupación óptima en cuanto a la $F$ discriminante (Pillais, Hotelling, y Wilks), pues si subíamos o bajábamos el número de clusters, empezaban a disminuir los coeficientes comprobados.

\begin{tabular}{|l|c|c|c|c|}
\hline Test Name & 3 & $\mathbf{4}$ & 5 & 6 \\
\hline Pillais & 25 & $\mathbf{3 0}$ & 16 & 14 \\
Hotelling & 62 & $\mathbf{5 8}$ & 63 & 42 \\
Wilks & 41 & $\mathbf{4 4}$ & 36 & 28 \\
\hline
\end{tabular}

* Pillais, Hotelling y Wilks son tests multivariados de significación de la F, que utilizan distintos tratamientos: transformando la F estadística (Pillais), basado en la suma de los valores obtenidos en los análisis discriminante, manova y factorial (Hotelling) y utilizando los valores de Lambda para observar las diferencias de medias (Wilks).

De esta forma encontramos, en la muestra analizada, cuatro subtipos de deficientes mentales, basados en la motricidad gruesa. 
3. Para facilitar la interpretación de las posibles soluciones, se trazaron los perfiles de desarrollo motor obtenidos por cada grupo, utilizando para ello las medias obtenidas en cada subtest del Bruininks-Ozeretsky (ver fig. 1).

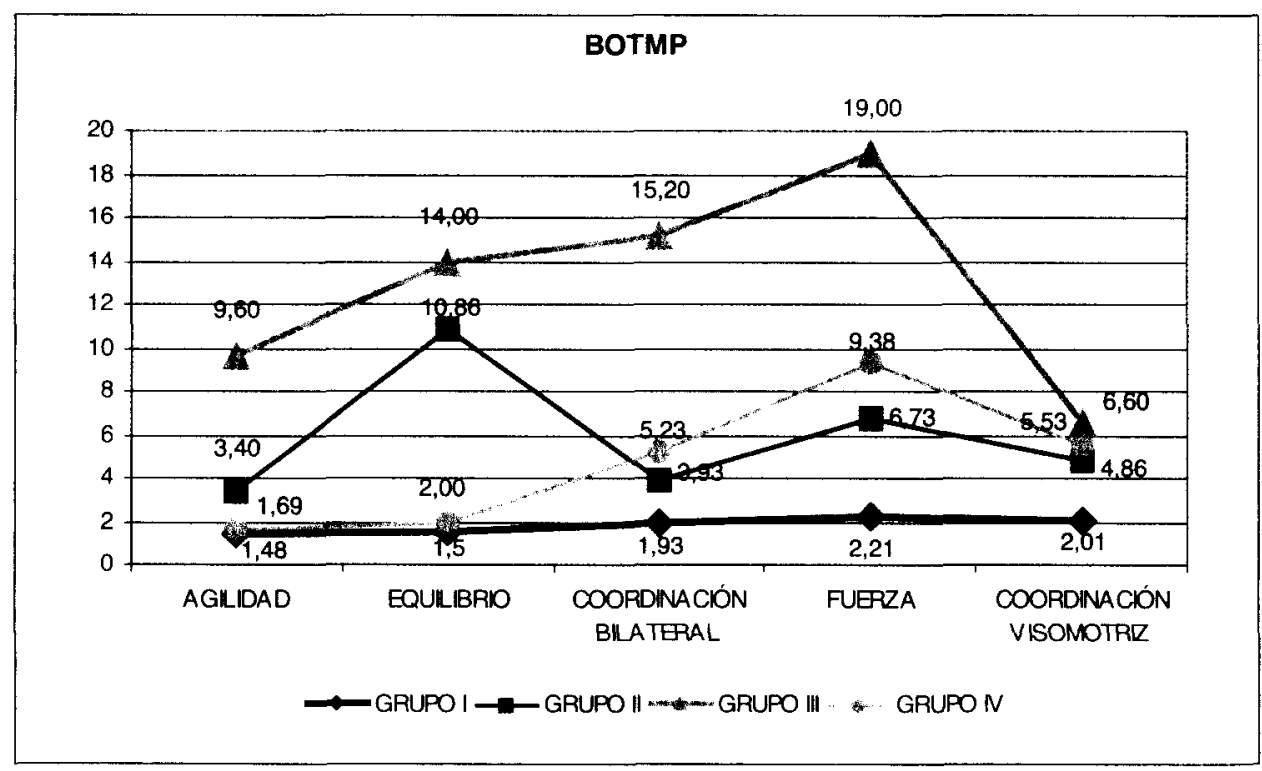

Los cuatro subtipos obtenidos presentan las siguientes características:

\section{Subtipo 1: Problemas motores generalizados}

Este primer grupo acoge al $65^{\prime} 98 \%(n=64)$ de la muestra de chicos/as con deficiencia mental. Este subtipo se caracteriza por tener unos resultados bastante homogéneos en todas las variables del Indice de funcionamiento del desarrollo motor grueso, muy por debajo de la media de la normalidad, es decir, presenta problemas motores generalizados.

\section{Subtipo 2: «Buen equilibrio»}

Este cluster acoge al $15^{\prime} 46 \%$ de la muestra $(n=15)$. Se caracteriza por una pobre ejecución en los subtests motores gruesos, sobresaliendo con diferencia la puntuación obtenida en el equilibrio, aun siendo también ésta baja. Hay otro subtest, el que mide la fuerza, que tiene una ligera tendencia a despuntar, pero, no obstante, sigue siendo muy bajo.

\section{Subtipo 3:Sin problemas motores}

Un grupo de cinco alumnos $\left(5^{\prime} 16 \%\right)$ demostraron tener un nivel de ejecución motriz gruesa dentro de la normalidad. Sin embargo, en lo que respecta a coordinación viso-motriz, sus resultados descendían notablemente. 


\section{Subtipo 4: «Fuerza destacable»}

Este último grupo representa un $13^{\prime} 40 \%$ de la muestra analizada ( $\mathrm{n}=$ 13). Aunque presenta unos resultados por debajo de la media, tiene como característica más sobresaliente la puntuación obtenida en el subtest que mide la fuerza. Del resto de los subtests hay que destacar que las dos coordinaciones (bilateral y viso-motriz) puntúan más alto que el equilibrio y la agilidad.

4. Se utilizó el análisis de varianza para observar si existían diferencias significativas entre las medias obtenidas en las distintas agrupaciones o clusters. Es decir, que las diferencias intragrupos eran mínimas y las diferencias intergrupos máximas, con una probabilidad de que esto sucediera de 0 '0000.

Tras el empleo de dicho análisis se observó que los cuatro perfiles tenían diferencias significativas entre las medias obtenidas en los cinco subtests que componen el Îndice de desarrollo motor grueso del Bruininks-Ozeretsky; «en concreto»:

4.1. En la variable agilidad, en los grupos $1\left(\mathrm{x}=1\right.$ '48) y $4\left(\mathrm{x}=1^{\prime} 69\right) \operatorname{los}$ resultados obtenidos son similares (no existen diferencias significativas entre las medias de ambos grupos), mientras que no sucede lo mismo con el $2\left(x=3^{\prime} 40\right)$ y el $3\left(x=9^{\prime} 60\right)$.

4.2. En equilibrio, de nuevo son los grupos $1\left(x=1^{\prime} 5\right)$ y $4\left(x=2^{\prime} 00\right)$ los que no se diferencian significativamente, mientras que los resultados obtenidos por el grupo $2\left(x=33^{\prime} 40\right)$ es bueno (recordemos que se caracteriza por su "buen equilibrio") y el grupo $3\left(x=14^{\prime} 00\right)$ tiene unas puntuaciones dentro de la normalidad.

4.3. En el subtest coordinación bilateral todos los grupos se diferencian significativamente.

$$
(F=62.3729 \quad P=.0000)
$$

4.4. Como en el caso anterior, la fuerza es una variable que distingue perfectamente a los cuatro grupos.

$$
(\mathrm{F}=110.4599 \quad \mathrm{P}=.0000)
$$

4.5. De nuevo, los resultados obtenidos por los cuatro grupos en la variable coordinación visomotriz son diferentes significativamente.

$$
(\mathrm{F}=14.7970 \quad \mathrm{P}=.0000)
$$




\section{Existe relación entre el nivel de desarrollo motor grueso y el grado de Deficiencia Mental}

Conforme el grado de deficiencia es mayor, suelen aparecer un mayor número de alteraciones anatómico-funcionales que inciden directamente en la presencia de trastornos y/o alteraciones en las habilidades motrices gruesas. (Rarick, 1973; Cratty, 1982; Molina de Costallat, 1983; Ruiz Pérez, 1986; Bucher, 1995; Toro y Zarco, 1995).

Nos interesa saber si, en nuestra muestra de deficientes, el nivel de desarrollo motor guarda alguna relación con las pruebas utilizadas habitualmente para medir el nivel intelectual (WISC y McCarthy), esperándose que cuanto menor sea éste, hay un mayor número de alteraciones en la motricidad gruesa.

En este caso vamos a plantear la siguiente hipótesis operativa:

«El grado de Deficiencia Mental se relaciona (correlaciona) con el nivel de desarrollo motor grueso alcanzado».

A la que corresponden las siguientes hipótesis estadísticas:

Ho: $\rho 0=0$; no existe relación entre dichas variables.

$H 1: \rho 1 \neq 0$; existe relación entre dichas variables.

Para validar esta hipótesis, con los resultados obtenidos en los subtests del BOTMP, WISC-R y MSCA, se utilizó una correlación parcial controlada por la edad, para evitar posibles correlaciones espúreas debidas al desarrollo de los individuos (Crowe, 1987; Muñoz Ruata, 1990).

En primer lugar, merece la pena destacar que las correlaciones obtenidas entre los cocientes de la "Escala McCarthy de Aptitudes y Psicomotricidad» (MSCA) y el "Test de Bruininks-Ozeretsky para medir la eficacia motriz» (BOTMP), en general, han sido significativas; no se puede decir lo mismo de las habidas entre la "Escala Wechsler de Inteligencia para niños-Revisada» (WISCR) y el «Test de Bruninks-Ozeretsky para medir la eficacia motriz» (BOTMP).

En concreto, todos los cocientes del MSCA (verbal, manipulativo, numérico y, por supuesto, el total) correlacionan significativamente con cuatro de los cinco subtests que componen el Índice de Desarrollo Motor Grueso del BOTMP. Sólo en el caso de la Coordinación Visomotriz, no se dan dichas significaciones.

\begin{tabular}{|c|c|c|c|c|c|}
\hline & AGITIP & EQUITIP & COBILTIP & FUERTIP & COVMTIP \\
\hline $\begin{array}{l}\text { MSCA } \\
\text { (CIT) }\end{array}$ & $\begin{array}{c}.3993 \\
(54) \\
\mathbf{P}=.002\end{array}$ & $\begin{array}{c}. \mathbf{3 8 9 1} \\
(54) \\
\mathbf{P}=. \mathbf{0 0 3}\end{array}$ & $\begin{array}{c}.3734 \\
(54) \\
P=.005\end{array}$ & $\begin{array}{c}. \mathbf{4 0 8 9} \\
(54) \\
\mathbf{P}=.002\end{array}$ & $\begin{array}{c}.1464 \\
(54) \\
\mathrm{P}=.282\end{array}$ \\
\hline
\end{tabular}




\begin{tabular}{|c|c|c|c|c|c|}
\hline & AGITIP & EQUITIP & COBILTIP & FUERTIP & COVMTIP \\
\hline $\begin{array}{l}\text { WISC } \\
\text { (CIT) }\end{array}$ & $\begin{array}{c}.1939 \\
(30) \\
\mathrm{P}=.288\end{array}$ & $\begin{array}{c}.1420 \\
(30) \\
\mathrm{P}=.438\end{array}$ & $\begin{array}{c}.2577 \\
(30) \\
\mathrm{P}=.155\end{array}$ & $\begin{array}{c}.2607 \\
(30) \\
\mathrm{P}=.150\end{array}$ & $\begin{array}{c}.0758 \\
(30) \\
\mathrm{P}=.680\end{array}$ \\
\hline
\end{tabular}

Mientras que sólo el Cociente Manipulativo del WISC-R correlaciona significativamente con dos subtests del BOTMP (Coordinación Bilateral y Fuerza).

\begin{tabular}{|c|c|c|c|c|c|}
\hline & AGITIP & EQUITIP & COBILTIP & FUERTIP & COVMTIP \\
\hline WISC & $\begin{array}{c}.2342 \\
(30)\end{array}$ & $\begin{array}{c}.2646 \\
(30)\end{array}$ & $\begin{array}{c}\mathbf{3 8 9 7} \\
(30)\end{array}$ & $\begin{array}{c}\mathbf{. 3 4 2 0} \\
(30)\end{array}$ & $\begin{array}{c}.2536 \\
(30)\end{array}$ \\
(CIM) & $\begin{array}{l}\mathbf{P = . 0 2 7} \\
\mathbf{P = . 1 9 7}\end{array}$ & $\mathbf{P = . 1 4 3}$ & $\mathbf{P = 5 5}$ & $\mathrm{P}=.161$ \\
\hline
\end{tabular}

Puesto que la MSCA está considerada como una escala útil para evaluar las potencialidades de los niños con Deficiencia Mental, y que el WISC-R se utiliza para aquellos individuos que poseen un mayor nivel intelectual, podemos constatar estadísticamente que, efectivamente, existe un mayor número de correlaciones significativas con las habilidades motrices gruesas conforme el grado de deficiencia mental es mayor.

Si analizamos las correlaciones obtenidas entre los subtests que componen las baterías utilizadas para este contraste de hipótesis, observamos que:

1. La coordinación bilateral del BOTMP correlaciona con:

- Construcción de cubos (MSCA)

$$
\mathrm{R}=.3157 \quad \mathrm{P}=.015
$$

- Rompecabezas (MSCA)

$$
\mathrm{R}=.3443 \quad \mathrm{P}=.008
$$

- Secuencia de golpeo (MSCA)

$$
\mathrm{R}=.3582 \quad \mathrm{P}=.0000
$$

- Coordinación de piernas (MSCA)

$$
\mathrm{R}=.3301 \quad \mathrm{P}=.013
$$


- Dibujo (MSCA)

$$
\mathrm{R}=.2605 \quad \mathrm{P}=.046
$$

- Recuento (MSCA)

$$
\mathrm{R}=.4022 \quad \mathrm{P}=.002
$$

- Cubos (WISC)

$$
\mathrm{R}=.3901 \quad \mathrm{P}=.027
$$

En algunos casos las pruebas son muy similares, por lo que es lógico que existan esas correlaciones, como es en el caso de la secuencia de golpeo y de la coordinación de piernas.

Por otro lado, Williams (1983) ya analizó la relación existente entre el desarrollo de la coordinación entre ambas manos (bilateral) y otros aspectos de la motricidad manual como:

- Manipulación de objetos (construcción de Cubos y/o Rompecabezas);

- Copia de formas o diseños (Dibujo);

- y Movimientos, que, aunque no tienen que ver con la motricidad manual, se consideran de motricidad fina, como son los movimientos de alternancia de talón-punta de los pies y de repiqueteo de los dedos (secuencia de golpeo), que, como hemos podido observar, también se dan en nuestra muestra.

2. La Agilidad del BOTMP correlaciona con la Coordinación de Piernas del MSCA $(R=.2397 \quad \mathrm{P}=.075)$, confirmándose los estudios de Borms (1986) que analizó la relación entre la mejora de la agilidad y el desarrollo del Sistema Nervioso Central y de la coordinación.

También otros autores (Espenschade, 1980; Zaichowsky, 1980; Williams, 1983; y Wickstrom, 1983) estudiaron la estructura de la carrera (agilidad) semejante a la marcha pues existen ajustes neuromusculares semejantes (coordinación de piernas), aunque para la velocidad se requiere un ajuste más rápido de los músculos agonistas y antagonistas de forma coordinada.

3. La Agilidad del BOTMP también correlaciona con la Memoria Pictórica del MSCA $(\mathrm{R}=.2827 \mathrm{P}=.035)$ y con Historietas del WISC $(\mathrm{R}=.3981 \mathrm{P}$ $=.024)$, confirmándose así los estudios de Chi (1976) que analizó la relación entre los déficits en las estrategias y en la velocidad de procesamiento y las diferencias en la realización y retención de tareas motrices. 
4. Se ha observado la relación existente entre la Fuerza y el Equilibrio del BOTMP y la Coordinación de Piernas del MSCA.
$\mathrm{R}=.2646$
$\mathrm{P}=.049$
(Fuerza)
$R=.3491$
$\mathbf{P}=.008$
(Equilibrio)

Diversos trabajos realizados (Espenschade, 1980; Cratty, 1982, 1986) han mostrado que la marcha (coordinación de piernas y pies), para que sea más armoniosa, necesita una mayor fuerza y un mayor desarrollo de los mecanismos sensoriomotores que permiten mejor equilibración y mayor coordinación neuromuscular.

La falta de fuerza en las piernas (fuente de impulso y de traslación por el espacio y medio de soporte) y la falta de estabilidad provoca en el niño una falta de coordinación y soltura, y hace que sus movimientos sean espasmódicos, rígidos y agitados. (Cratty, 1982).

5. Al valorar la estructura madura de cualquier movimiento que implique coordinación visomotriz (recepción, lanzamiento, bote y tiro de precisión), podemos destacar la necesidad de una posición correcta del cuerpo, una buena persecución visual que realizan los ojos, una buena coordinación de brazos y manos y una posición equilibrada y estable de los pies. (Wellman, 1931; Cratty, 1982; Williams, 1983; Wickstrom, 1983). Por lo que no es de extrañar que hayamos obtenido correlaciones significativas entre la Coordinación de Brazos del MSCA y la Coordinación Visomotriz del BOTMP.
$(\mathrm{R}=.5359$
$\mathrm{P}=.000)$

6. Como hemos comentado anteriormente, la falta de fuerza y la falta de equilibrio provocan en los niños problemas de coordinación, lo que hace que sus movimientos sean espasmódicos, rígidos y agitados. (Cratty, 1982) De esta manera podríamos justificar la correlación hallada entre el Equilibrio del BOTMP y la prueba de Claves del WISC.
$(\mathrm{R}=.3455$
$\mathrm{P}=.053)$

Los resultados obtenidos coinciden con los trabajos de: Cratty (1982), Molina de Costallat (1983) Arnaiz (1983), González (1985), Crowe (1987) Oña Sicilia (1987), Berges (1990), Moral (1994) y Rosa Neto (1996); aunque hay que tener en cuenta las distintas características de los mismos. Es decir, conforme el grado de deficiencia es mayor, suelen aparecer un mayor número de trastornos y/o alteraciones en las habilidades motrices. 


\section{CONCLUSIONES}

Tras el análisis de los resultados obtenidos, se rechazan las hipótesis nulas presentadas y se aceptan las hipótesis alternativas, por lo que podemos decir que:

1. Es posible identificar subtipos de sujetos con deficiencia mental según el rendimiento en habilidades motrices gruesas.

2. Existe relación entre el nivel dedesarrollo motor grueso y el grado de Deficiencia Mental.

Podemos añadir también que:

1. Para la evaluación de las habilidades motrices gruesas se puede utilizar el Test de Bruininks-Ozeretsky para medir la eficacia motriz, batería que no ha sido realizada para esta población (como es el caso de la batería de Picq y Vayer), ni adaptada para poblaciones con esta deficiencia (como el Test AAHPER para medir el rendimiento físico).

2. Los resultados obtenidos apuntan a que la deficiencia mental no es un todo homogéneo, sino que es posible identificar diversos perfiles en el ámbito del desarrollo motor grueso. Ello apoyaría la necesidad de una evaluación psicopedagógica y de una intervención específica del deficiente mental.

3. Se han podido identificar cuatro subtipos de desarrollo motor, cuyas diferencias son estadísticamente muy significativas. Dichas diferencias deberían tenerse en cuenta a la hora de realizar las adaptaciones curriculares pertinentes en el área de Educación Física.

4. Los alumnos que componen el subtipo 1 son pobres en todas las funciones motrices gruesas y necesitan programas paliativos específicamente diseñados para ellos.

Además de intervenciones motrices diseñadas para mejorar el funcionamiento motor, un programa especial para este conjunto de deficientes debería considerar el progreso en relación al bienestar socioemocional, puesto que los niños deficientes mentales que además son torpes y desmañados físicamente tienden a tener una autoestima más baja que la de aquellos que no lo son.

Asimismo, consideramos necesario realizar un cuidadoso análisis de las tareas que permitiesen a los niños, con pobres funciones motrices gruesas, experimentar éxitos motrices y recibir refuerzo positivos, tanto externos como internos, confirmando, de esta manera, los resultados de anteriores investigaciones como los de Gubbay (1975), Shaw (1982), Reid (1987) y Montero (1993). 
[Se ha demostrado que las combinaciones de experiencias exitosas y "feedback» positivo han facilitado la motivación intrínseca y la percepción de competencia (Montero, 1993) y han mejorado las habilidades de movimiento (Vallerand y Reid, 1984)].

5. El subtipo 2 está caracterizado por un buen equilibrio y resultados pobres en las otras variables motrices gruesas, aunque despunta ligeramente la fuerza.

Puesto que es posible que ambas variables no puedan compensar el resto de dificultades motrices, los alumnos de este grupo deberían ser tratados de manera similar a los del subtipo 1. Es decir, con el fin de evitar reacciones emocionales negativas, se debería focalizar el trabajo de esta área en las variables más fuertes (en este caso el equilibrio y la fuerza) y prestar una atención indirecta a las áreas más débiles, siguiendo los trabajos realizados por Coplin y Morgan $(1988,1990)$.

Sería conveniente que los aspectos motrices débiles (coordinación bilateral, coordinación visomotriz y agilidad) fueran tratados o rehabilitados desde otras áreas como la fisioterapia, la psicomotricidad, la danzaterapia o en actividades realizadas en el medio acuático.

6. Los alumnos del subtipo 3 no requieren programas compensatorios y/o adaptaciones curriculares, ya que no poseen disfunciones severas en la motricidad gruesa. En cualquier caso, las actividades físicas y, más concretamente, los deportes pueden ser un vehículo ideal para que ganen en autoconfianza y aceptación social (Martín y Martín, 1988), lo que les facilitaría su integración en la sociedad de la que ellos se sienten excluídos.

7. El subtipo 4 está caracterizado por habilidades motrices gruesas pobres y resultados en la fuerza cercano a la media.

Se debería tratar a estos alumnos de manera similar a los del subtipo 2, para evitar reacciones emocionales negativas, es decir, se debería animar a estos chicos a realizar actividades de fuerza (área que despunta entre todas las variables motrices) y tratar con atención indirecta (fisioterapia, psicomotricidad, terapias de integración sensorial, etc...) a aquellos aspectos motrices débiles.

8. Hay una relación significativa entre el nivel de desarrollo motor grueso y el grado de desarrollo intelectual alcanzado por los deficientes mentales, estimado mediante las medidas habitualmente aceptadas de nivel intelectual (MSCA y WISC-R).

El trabajo ha permitido comprobar que, conforme el grado de deficiencia es mayor, suele existir mayor correlación con el área motriz 
gruesa y aparecen un mayor número de trastornos y/o alteraciones en las habilidades motrices. (Recordemos que el subtipo 1 que no sólo presenta problemas motores generalizados, sino que además los resultados en las pruebas del BOTMP son los más bajos de los obtenidos por todas las agrupaciones, se caracteriza por tener el menor cociente intelectual total de todos los grupos).

9. Los resultados apuntan que la MSCA es un buen predictor del nivel de desarrollo motor grueso y mejor que el WISC-R, pues existe un mayor número de correlaciones significativas entre las variables medidas por la MSCA y las medidas por el BOTMP.

10. Las variables visoconstructivas también han sido estudiadas en relación con las variables motrices. Así, comprobamos que la construcción de cubos, rompecabezas, dibujos e historietas (MSCA y WISC-R) son las variables más relacionadas con la coordinación bilateral y la agilidad.

11. Las pruebas de memoria, medidas por los tests psicométricos (como la memoria pictórica del MSCA), también guardan relación con el nivel de desarrollo motor grueso.

12. Se han podido identificar perfiles "cognitivos» en función de los perfiles motrices obtenidos en este estudio, de manera que nos proporcionen la información necesaria para elaborar adaptaciones curriculares pertinentes.

13. El subtipo 1, además de caracterizarse por problemas motrices generalizados, tiene un grado de deficiencia mental de tipo moderado (CIT en el MSCA de 50'15 y en el WISC-R de 50'07) y presenta alteraciones en variables visoperceptivas.

Será necesario, por tanto, realizar adaptaciones no sólo en la metodología (estrategias de motivación y refuerzos variados), sino en el medio de aprendizaje. Es decir, eliminaremos las fuentes de distracción y utilizaremos rituales que proporcionen ayuda para memorizar las secuencias motrices a trabajar, y simplificaremos las percepciones visuales a través de colores vivos, diferentes tamaños y formas para identificar movimientos, circuitos y/o estaciones a realizar.

14. El subtipo 2 está caracterizado por un buen equilibrio, dentro de unos rendimientos bajos en las habilidades motrices gruesas. Tiene un grado de deficiencia mental de tipo ligero (CIT de 65). Aunque existen diferencias entre las medias obtenidas en la MSCA $\left(65^{\prime} 20\right)$ y el WISC-R (50'20), como el mayor número de individuos han sido medidos con la MSCA, hemos tenido en cuenta la puntuación obtenida en esta batería; es decir, que este perfil se dará en sujetos con Deficiencia Mental ligera. 
Las adaptaciones curriculares individuales necesarias para este grupo de alumnos se enfocarán en los siguientes aspectos:

- Adaptaciones en la metodología:

- estrategias de motivación

- refuerzos variados

- actividades alternativas

- Adaptaciones pedagógicas:

- apoyos verbales, visuales y manuales

- división del movimiento en secuencias

- Adaptaciones del medio de aprendizaje:

- eliminar fuentes de distracción

- utilizar rituales

- Simplificaciones:

- de las percepciones

- de los gestos.

15. Los alumnos del subtipo 3 que componen este grupo se caracterizan por estar dentro de la normalidad en cuanto a habilidades motrices gruesas se refiere.

No es de extrañar este hecho, puesto que la media obtenida en el CI total, calculado por el WISC-R, es de 70'50, es decir, son personas «borderline», a caballo entre la normalidad y la deficiencia mental ligera.

Como son individuos que, en la mayoría de los casos, han sufrido privación socioafectiva en la primera infancia, se sienten poco integrados en la sociedad. Por ello creemos que sería conveniente utilizar esta área motriz, en la que destacan notablemente sobre otras áreas pedagógicas, como vehículo para lograr su integración social.

16. El subtipo 4 se caracteriza por una fuerza destacable, un grado de deficiencia mental de tipo ligero (CIT en la MSCA de 60'25 y en el WISC-R de 62'40) y alteraciones en variables visoperceptivas. 
Por sus características parecidas, se realizarán las adaptaciones curriculares propuestas en el punto 14 para el subtipo 2, teniendo en cuenta que se deberá rehabilitar aquellas áreas motrices cuyos resultados difieren significativamente con los obtenidos por dicho grupo.

Por todo ello, consideramos que se debe diseñar programas específicos que intenten paliar los efectos de los trastornos y alteraciones motrices gruesas analizados en este estudio, comprobando, posteriormente, su idoneidad para mejorar los resultados obtenidos en esta área, así como realizar las adaptaciones curriculares del área de Educación. Física, basándose en los perfiles de desarrollo motor grueso obtenidos.

\section{BIBLIOGRAFÍA}

ARIEL, H. (1990). "Importancia de la educación física en el desarrollo de la percepción de los niños discapacitados mentales», en ANDE, n. ${ }^{\circ}$ 17, pp. 8-12.

ARNAIZ, P. (1983). «Estudio correlacional entre el desarrollo de las funciones psíquicas y el desarrollo de las funciones motoras. Directrices para la educación psicomotriz de los deficientes mentales", en Anales de Pedagogia, 2, 327-343.

ARNAIZ, P., y LOZANO, J. (1996). Proyecto curricular para la diversidad. Psicomotricidad y lectoescritura, CCS, Madrid.

BALIUS, R. (1989). "Repercusión del ejercicio físico y el deporte sobre el aparato locomotor", en Jano, Monografías médicas, vol. $3, \mathrm{n} .^{\circ} 8,55-58$.

BERGES, J. (1990). "Los trastornos psicomotores del niño", en LEBOVICI, S., DIATKINE, R., y SOULE, M., Tratado de psiquiatría del niño y del adolescente, Tomo V, Biblioteca Nueva, Madrid.

BLÁZQUEZ SÁNCHEZ, D. (1990), Evaluar en Educación Física, INDE, Barcelona.

BONDA, P.F. (1986). "Criteri e strategie generali di intervento", en Risposte, n. ${ }^{\circ}$, pp. 19-24.
BRANFORD, D. (1995). "Dyskinetic movements in a population of people with learning disabilities», en British joumal of developmental disabilities, vol. 41, n. $^{\circ} 80$, pp. 23-32.

BROADHEAD, G.D., CHURCH, G.E. (1982). "Discriminant analysis of gross and fine motor proficiency data", en Perceptual Motor Skills, Oct. 55 (2), 547-552.

BRUININKS-OSERETSKI TEST (1978). American Guidance Service, Circle Pines, Minesota 55014.

BUCHER, H. (1976). Trastornos psicomotores en el niño, Masson, Barcelona, 1988.

BULBENA, A. (1985). "Psicopatología de la Psicomotricidad", en VALLEJO, J., Introducción a la Psicopatología y a la Psiquiatría, Salvat, Barcelona.

CAMUS, J.L. (1987). La práctica psicomotriz en el niño poco hábil, Marfil, Alicante.

CARON, F., y BOUFFARD, M. (1990). "Education motrice», en L'intervention en deficience mental, 2, Pierre Mardaga, Bruselas.

CASTILLO, N. Del (1987). "Características del aprendizaje motor-perceptual en un grupo de jóvenes retrasados mentales", en Boletín de psicologia, vol. $10, \mathrm{n}^{\circ} 1$, pp. $38-57$. 
CROWE, T.K. (1987). "The relationship between the Bayley scales of infant development and preschool gross motor and cognitive performance", en American Joumal of occupational therapy, vol. 41, n. ${ }^{\circ} 6$, pp. 374-378.

DROWATZKY, J.N. (1973). Educación Física para niños deficientes mentales. Panamericana, Buenos Aires.

DSM-IV (1995). Manual Diagnóstico y estadistico de los trastomos mentales, Masson, Barcelona.

FETZ, F., y KORNEXL, E. (1976). Test deportivo-motores, Kapelusz, Buenos Aires.

FIERRO, A. (1987). «Desarrollo cognitivo, intervención e integración educativa en los deficientes mentales", en Revista de Educación, n. ${ }^{\circ}$ extraordinario, pp. 105-131.

GAVIRIA SOTO, J.L. (1986). «El enfoque diferente en la investigación pedagógica", en Bordón, n. ${ }^{0} 262$, marzoabril.

GELOF, M. (1963). "Comparison of systems of classification relating degree of retardation to measured intelligence» en American Journal of Mental Deficiency. Vol. 68, pp. 299-301.

JANSMA, P. (1988). «A fitness assessment system for individuals with severe mental retardation", en Adapted physical activity quarterly, vol. 5, n. 3 , pp. 223-232.

LAHTINEN, U. (1977). Physical fitness and psychomotor performance of mentally retraded children, International Symposium of APA, Tomo 2, Quebec, Canadá.

LINARES, P.L. (1994). "Deficiencia mental: psicopedagogía y motricidad especial", en Polibea, n. ${ }^{\circ} 30, \mathrm{pp}$. 8-16.

LINARES, P.L. (1995). «Educación física en un centro ocupacional de adultos con deficiencia mental", en Polibea, n. 35 , pp. 4-10.

LÓPEZ-BARAJAS, E., LÓPEZ LÓPEZ, E., PÉREZ JUSTE, R. (1987). Pedagogia Experimental I, Vol. I y II, UNED, Madrid.
LUCKASSON, R., COULTER, D.L., POLLOWAY, E., REISS, S., SCHALLOCK, R.L., SNELL, M.E., SPITALNICK, D.M., y STARK, J.A. (1992). "Mental Retardation: Definition, Classification and Systems of supports", en American Association on Mental Retardation, Washington, D.C.

MARTÍN, F. (1990). La Educación Física del deficiente mental, Gymnos, Madrid.

MIYAHARA, M. (1994). "Subtypes of students with learning disabilities based upon gross motor functions", en Adapted physical activity quarterly, vol. $11, n{ }^{\circ} 4$, pp. $368-382$.

MOLINA DE COSTALLAT, D. (1986). Psicomotricidad II. El niño deficiente mental y psicomotor, Losada, Buenos Aires.

PALLISERA, M. (1995). «Aplicación cuasiexperimental de un programa de intervención en las habilidades perceptivo-motrices en personas adultas con disminución psíquica», en Revista de educación especial, n. ${ }^{\circ} 19$, pp. 37-53.

PANERAI, S. (1986). "Programmi de intervento per promuovere l'acquisizione di alcune abilitá nell'area psicomotoria, dirette a bambini con ritardo mentale grave e profondo", en Quaderno Oasi, n. ${ }^{\circ} 11$, pp. 53-171.

ROSA NETO, F. (1996). Valoración del desarrollo motor y su correlación con los trastornos del aprendizaje, Tesis Doctoral no publicada. Universidad de Zaragoza.

RUIZ PÉREZ, L.M. (1986). Actividades físicas y deficiencia mental, Congreso Nacional de Educación Física, "La Educación Física Hoy», Barcelona.

SEEFELDT, V. (1981). "Revisión de las investigaciones publicadas sobre programas percepto-motores", en Siglo Cero, 78, 27-38.

SHERRILL, C. (1990). «La educación física adaptada como aspecto clave en la formación del profesorado especial para personas con retraso mental", en Temas clave en investigación del retraso mental, SIIS, Madrid. 
STONE R.K. (1989). "Prevalence of dyskinesia and related movement disorders in a developmentally disabled population", en Joumal of mental deficiency research, vol. 33, n. ${ }^{\circ}$, pp. 41-53.

ULRICH, D.A. (1989). "Assessing movement control in children with mental retardation: a generalizability analysis of observers", en American joumal of mental retardation, vol. $94, n{ }^{\circ} 2$, pp. 170-176.

ZIVIANI, J., POULSEN, A., O'BRIEN, A. (1982). "Correlation of the Bruininks-Oseretsky Test of Motor Proficiency with tha Southern California Sensory Integration Tests", en American Journal Occupational Therapy, Aug. 36 (8), 519-523.

\section{RESUMEN}

El propósito de este estudio ha sido identificar posibles subtipos de alumnos con Deficiencia Mental basados en funciones motrices gruesas. Se seleccionó un grupo de alumnos de un centro especifico de Deficiencia Mental, que no presentaron deficiencias motóricas asociadas, y se les administraron los subtests de motricidad gruesa del "Test Bruininks-Ozeretsky para medir la eficacia motriz".

Los cuatro subtipos resultantes mostraron diferentes perfiles de desarrollo motor.

A partir de estos resultados, se recomienda diseñar programas especificos que intenten paliar los trastornos motrices gruesos, analizados para cada uno de los subtipos hallados, y realizar adaptaciones curriculares pertinentes, teniendo en cuenta los perfiles motrices estudiados.

Palabras clave: Deficiencia Mental. Trastornos motrices gruesos. Test Bruininks-Ozeretsky.

\section{ABSTRACT}

The goal of this work was to identify some different cathegories of mental retarded people, depending on their gross motor functioning. A group of mental retarded people was selected from a special education school. None of theese students presented any associated motor deficiency. The gross motor subtests from the Bruininks-Ozeretsky test were administered in order to evaluate the motor eficiency of these mental retarded people. The resulting four cathegories showed different motor development profiles. From theese results it's recommended to design curricula-based intended to amiliorate the specific gross motor deficits of each group and making didactical schedules taking into account the motor profiles studied. 